\title{
The Proportional Hazards Model with Linearly Time-dependent Covariates and Interval-censored Data
}

\author{
Qiqing Yu and Qinggang Diao \\ Department of Mathematical Sciences, SUNY, Binghamton, NY 13902, USA \\ Email: qyu@math.binghamton.edu
}

\begin{abstract}
The semi-parametric estimation under the proportional hazards ( $\mathrm{PH})$ model with a linearly time-dependent covariates and with interval-censored data has not been investigated before. The partial likelihood approach does not work and one has to use the generalized likelihood function (GLF). There is a challenge in this problem. The GLF must be in the form of the baseline hazard function, rather than the baseline survival function as in the $\mathrm{PH}$ model with time-independent covariates, and a feasible way to specify the hazard function is a piece-wise constant function. However, several naive ways do not yield a consistent estimator. We propose proper modifications of the GLF. Simulation results suggest that our method works. The generalization to other types of time-dependent covariates is also discussed.
\end{abstract}

Keywords: Cox's model, time-dependent covariates, modified likelihood function, semi-parametric MLE.

\section{Introduction}

We consider the semiparametric estimation problem under the proportional hazards $(\mathrm{PH})$ model with a special continuous time-dependent covariates and with interval-censored data. In this paper, we assume that the survival time $Y$ is continuous.

Interval-censored (IC) data are $\left(L_{i}, R_{i}\right), i=1, \ldots, n$, where the true survival time $Y_{i} \in\left(L_{i}, R_{i}\right]$. The proportional hazards model (Cox (1972)) specifies that a covariate vector $\mathbf{z}$ has a proportional effect on the hazard function of $Y$. This model provides powerful means for fitting failure time observations to a distribution free model and for estimating the risk for failure associated with a covariate vector $\mathbf{z}$.

For a continuous random variable $Y$, denote its cumulative distribution function (cdf) by $F_{Y}$, its survival function by $S_{Y}(t)=1-F_{Y}(t)$, its density function (df) by $f_{Y}(t)$, and its hazard function by $h_{Y}(t)=\frac{f_{Y}(t)}{S_{Y}(t)}$. We say that $(\mathbf{z}, Y)$ follows a PH model or Cox's regression model if the hazard of $Y \mid \mathbf{z}$ is

$$
h(t \mid \mathbf{z})=h_{o}(t) e^{\beta \mathbf{Z}}, \text { for } t<\tau,
$$

where $\beta \mathbf{z}=\beta^{\prime} \mathbf{z}, \beta^{\prime}$ is the transpose of the vector $\beta, \tau=\sup \left\{t: h_{o}(t)>0\right\}$, and $h_{o}$ is the hazard function of $Y \mid(\mathbf{z}=0)$. The PH model has been extended to time-dependent covariates, that is, $h(t \mid \mathbf{z})=$ $e^{\beta \mathbf{Z}(t)} h_{o}(t), t<\tau$, where the covariate $\mathbf{z}(t)$ is also a function of the time $t$. In Cox and Oakes (1984 p.113), two examples of such extension are given. The PH models in those examples can be written as the form $h(t \mid u)=\exp (u \beta g(t)) h_{o}(t), t<\tau$, where $u$ is a time-independent covariate, and $g(t)$ is a function of the time $t$. Two particular examples presented there are $g(t)=\mathbf{1}(t \geq a)$ and $g(t)=(t-a) \mathbf{1}(t \geq a)$, respectively, where $\mathbf{1}(A)$ is the indicator function of the event $A$. The first example is a special case of the piecewise PH model (PWPH model) and the second one is called the PH model with linearly time-dependent covariates (LDPH model). Therneau and Grambsch (2000) provide a computer program for computing the partial likelihood estimator under the $\mathrm{PH}$ model with such $z_{i}(t)$ and with right-censored data.

The TDCPH model has been commonly used for right-censored (RC) data (see, for instance, Zhou (2001), Leffondre et al. (2003), Platt et al. (2004), Zhang and Huang (2006), Stephan and Michael (2007), Masaaki and Masato (2009) and Leffondre et al. (2010)). The semi-parametric estimation with IC data under the PWPH model is studied by Wong et al. (2018). However, the semi-parametric estimation with IC data under the LDPH model has not been studied so far. 
In this paper we consider the LDPH model, that is,

$$
\mathbf{z}_{i}(t)=\mathbf{u}_{i} *(t-a) \mathbf{1}(t \geq a), \text { where } \mathbf{u}_{i} \text { is a time-independent covariate vector }
$$

and $a$ is a real number. This covariate is very typical and shares the light on how to estimate under the TDCPH model with IC data and with other types of covariates.

We shall assume that the hazard function $h_{o}$ is unknown. Under such semi-parametric set-up, the typical estimation approach for right-censored data is the partial likelihood estimation. This approach simplifies the estimation procedure by estimating $\beta$ alone without estimating the baseline hazard function $h_{o}$ in the same time. Moreover, the properties of the partial likelihood MLE are quite similar to those of the generalized likelihood MLE. However, it is well known that this approach only works for right-censored data, but does not work for IC data (see, for example, Wong and Yu (2012)). Since we are dealing with IC data, we shall study the generalized likelihood estimation procedure in this paper.

For the covariate defined in (1.2), there are several theoretical issues to be settled. First, even if the parameter $\beta \in(-\infty, \infty), \beta$ may not be identifiable if the support set (of the observable random vector) is discrete (to be defined in Section 2). This is quite different from the case of the PH model with time-independent covariates, under which $\beta$ is identifiable even if the support set contains only one point. It is also quite different from the case of the $\mathrm{PH}$ model with the time-dependent covariate of the form $z(t)=u \mathbf{1}(t \geq a)$, under which $\beta$ is identifiable if the support set contains at least two points in $[a, \infty)$. The identifiability condition specifies the necessary condition under which a consistent estimator of $\beta$ is possible and also gives a guideline for the set-up of simulation studies.

Secondly, unlike the PH model with time-independent covariates, the generalized likelihood function needs to be modified, as it must be in the form of hazard functions under the semi-parametric set-up in (1.2). Otherwise, there is no consistent estimator of $\beta$. If the covariate $\mathbf{u}_{j}$ in (1.2) takes on finitely many values, there is a naive non-parametric estimator of $\beta$, called the generalized maximum likelihood estimator (GMLE). However, it is not efficient. As explained in Section 3, several naive modifications on the generalized likelihood function do not lead to consistent estimators. We propose a proper modification of the generalized likelihood to get a semi-parametric MLE (SMLE) of $\beta$ in this paper (see Remarks 2 and (3.3)). Our simulation studies suggest that the SMLE of $\beta$ is consistent and is more efficient than the GMLE.

We study the identifiability condition in Section 2. We study how to modify the generalized likelihood function for deriving the SMLE in Section 3. We also introduce the algorithm for obtaining the GMLE if the covariate takes on finitely many values. Simulation results for comparing the SMLE and the GMLE are presented in Section 4. The generalization to other types of time-dependent covariates is discussed in Section 5.

\section{The Models and the Identifiability Condition}

Under interval censoring without covariates, the observable random vector is $(L, R)$, where $L \leq Y \leq R$. The standard IC model that does not involve exact observations is the mixed case IC model (see Schick and $\mathrm{Yu}(2000)$ ). Its simplest special case is the Case 2 model (see Groeneboom and Wellner (1992)):

(1) The random vector $(U, V)$ and $(Y, u)$ are independent,

(2) $(L, R)=(-\infty, U) \mathbf{1}(Y \leq U)+(U, V) \mathbf{1}(Y \in(U, V])+(V, \infty) \mathbf{1}(Y>V)$.

The common IC model that involves exact observations is the double censorship model (see Turnbull (1976)). In this section we assume that $(Y, \mathbf{u})$ are from the PH model as in $(1.1)$, with $\mathbf{z}=\mathbf{u} \cdot(t-a) \mathbf{1}(t \geq a)$, $Y$ is continuous and is subject to interval censoring, and, $\mathbf{u}$ is a non-trivial time-independent random variable (vector). Interval-censored regression data are denoted by $\left(L_{i}, R_{i}, \mathbf{u}_{i}\right)$, where $L_{i}$ and $R_{i}$ are the endpoints of the interval $I_{i}$ and $Y_{i} \in I_{i}$. The generalized likelihood function with IC data $\left(L_{i}, R_{i}\right)$ 's is given by

$$
\mathcal{L}_{*}=\prod_{i=1}^{n} \mu_{S(\cdot \mid \cdot)}\left(I_{i}\right), \text { where } \mu_{S}\left(I_{i}\right)= \begin{cases}S\left(L_{i}\right)-S\left(R_{i}\right) & \text { if } L_{i}<R_{i} \\ S\left(L_{i}-\right)-S\left(L_{i}\right) & \text { if } L_{i}=R_{i}\end{cases}
$$




\subsection{General Forms of the Survival Functions}

Since the generalized likelihood (2.1) depends on the survival function $S(t \mid \mathbf{z}(t)$ ), we shall discuss the general form of $S(t \mid \mathbf{z}(t))$.

Theorem 1. Assume that $S(t \mid \mathbf{z}(t))$ satisfies the PH model and is absolutely continuous, and $\mathbf{z}(t)$ is a time-dependent covariate. Then $S(t \mid \mathbf{z}(t))=\exp \left(-\int_{-\infty}^{t} e^{\beta \mathbf{Z}(x)} h_{o}(x) d x\right)$.

Corollary 1. Under the assumptions in Theorem 1 , if $\mathbf{z}(t)=(t-a) \mathbf{u} \mathbf{1}(t \geq a)$, then

$$
S(t \mid \mathbf{z}(t))= \begin{cases}S_{o}(t) & \text { if } t \leq a \text { or } \mathbf{u}=0 \\ S_{o}(a) \exp \left(-\int_{a}^{t} e^{\beta \mathbf{u}(x-a)} h_{o}(x) d x\right) & \text { if } t>a \text { and } \mathbf{u} \neq 0\end{cases}
$$

In our proposed estimation method, we shall make use of the special hazard function as follows.

$$
\text { For } t \geq a, h_{o}(t)= \begin{cases}h_{i} & \text { if } t \in\left[a_{i}, b_{i}\right), i \in\{1, \ldots, k\} \\ 0 & \text { otherwise }\end{cases}
$$

where $a \leq a_{1}<b_{1} \leq a_{2}<b_{2} \leq \cdots \leq a_{k}<b_{k} \leq a_{k+1}=\infty$. Then

$$
S(t \mid \mathbf{z}(t))= \begin{cases}S_{o}(t) & \text { if } t \leq a_{1} \text { or } \mathbf{u}=0 \\ S_{o}(a) \exp \left(-\frac{e^{-a \mathbf{u} \beta}}{\mathbf{u} \beta}\left[\sum_{i=1}^{j-1} h_{i}\left[e^{\mathbf{u} \beta b_{i}}-e^{\mathbf{u} \beta a_{i}}\right]\right)\right. & \\ \left.\left.+h_{j}\left[e^{\mathbf{u} \beta t}-e^{\mathbf{u} \beta a_{j}}\right]\right]\right) & \text { if } t \in\left[a_{j}, b_{j}\right) \text { and } j \leq k \\ S_{o}(a) \exp \left(-\frac{e^{-a} \mathbf{u}_{\beta}}{\mathbf{u} \beta} \sum_{i=1}^{j} h_{i}\left[e^{\mathbf{u} \beta b_{i}}-e^{\mathbf{u} \beta a_{i}}\right]\right) & \text { if } t \in\left[b_{j}, a_{j+1}\right) \text { and } j \leq k .\end{cases}
$$

In particular, if $b_{i} \approx a_{i}$ for all possible $i$,

$$
\begin{aligned}
S(t \mid \mathbf{z}(t)) & \approx S_{o}(a) \exp \left(-\sum_{i=1}^{j} h_{i} e^{\left(b_{i}-a\right) \mathbf{u} \beta}\left(b_{i}-a_{i}\right)\right) \\
& =S_{o}(a) \exp \left(-\sum_{i=1}^{j} h_{i} e^{\left(b_{i}-a\right) \mathbf{u} \beta}\left[\left(b_{i}-a_{i}\right)+o\left(b_{i}-a_{i}\right)\right]\right) \text { if } \mathbf{u} \neq 0, t=b_{j}, b_{i} \approx a_{i}, j \leq k
\end{aligned}
$$

\section{$2.2 \quad$ Identifiability Issue}

We assume that the $p \times 1$ covariate vector $\mathbf{u}$ takes at least $p$ linearly independent values. In particular in this section, without loss of generality (WLOG), we assume $p=1$ and $u \in\{0,1\}$. Hereafter, by abuse of notations, we write $S(t \mid u)=S(t \mid \mathbf{z}(t))$ and $h(t \mid u)=h_{o}(t) \exp (\beta(t-a) u \mathbf{1}(t \geq a))$. Since $h_{o}\left(\right.$ or $\left.f_{o}\right)$ can differ on a set $A$ satisfying $\int_{A} d S_{o}(t)=0$, we define $f_{o}(t)=\left\{\begin{array}{ll}-S_{o}^{\prime}(t) & \text { if } S_{o}^{\prime}(t) \text { exists } \\ 0 & \text { otherwise }\end{array}\right.$ for identifiability of $f_{o}$ and $h_{o}$. Let $\mathcal{S}_{F}$ be the support set of the random variable with the cdf $F$, in the sense that

$$
x \in \mathcal{S}_{F} \text { iff } F(x+\epsilon)-F(x-\epsilon)>0, \forall \epsilon>0 .
$$

It is worth mentioning that if the df $f_{X}$ of a random variable $X$ exists, then the cdf $F_{X}$ and the hazard function $h_{X}$ of $X$ are equivalent in the sense that $f_{X}$ yields $F_{X}, F_{X}$ yields $h_{X}$ and $h_{X}$ yields $f_{X}$. However, one of $f_{X}, S_{X}$ and $h_{X}$ is given on a subset $A$ of $\mathcal{S}_{F_{X}}$, then it is not always true that the other two functions are known on $A$. A counterexample can easily be found by setting $A=\{2\}$ and $X \sim \operatorname{Exp}(\mu)$, the exponential distribution with mean $\mu$.

Lemma 1. The survival function $S(t \mid u)$ is identifiable if $t \in \mathcal{S}_{F_{L}} \cup \mathcal{S}_{F_{R}}$.

Proof. We say that the parameter $\theta$ is identifiable in the sense that the values of the df $f(\cdot ; \theta)$ uniquely determines the parameter $\theta$. Under the set-up in this section, the parameter is $S(t \mid u)$, where $u$ is given and the $\mathrm{df}$ is the $\mathrm{df}$ of $(L, R)$ for the given $u$, say $g(l, r \mid u ; S(\cdot \mid \cdot))$. WLOG, we can assume the Case 2 model with the two follow-up times $U$ and $V$, such that $(Y, u) \perp(U, V)$. Then $\left(\mathcal{S}_{F_{L}} \cup \mathcal{S}_{F_{R}}\right) \cap(-\infty, \infty)=\mathcal{S}_{F_{U}} \cup \mathcal{S}_{F_{V}}$.

$$
g(l, r \mid u ; S(\cdot \mid \cdot))= \begin{cases}(1-S(r \mid u)) f_{U}(r) & \text { if } l=-\infty \text { and } f_{U}(r)>0 \\ (S(l \mid u)-S(r \mid u)) f_{U, V}(l, r) & \text { if } f_{U, V}(l, r)>0 \text { and }-\infty<l<r<\infty \\ S(l \mid u) f_{V}(l) & \text { if } f_{V}(l)>0 \text { and } r=\infty \\ 0 & \text { otherwise. }\end{cases}
$$


If $f_{U}(t)>0$ then $S(t \mid u)=1-g(-\infty, t \mid u ; S(\cdot \mid \cdot)) / f_{U}(t)$ is uniquely determined by $g$. If $f_{V}(t)>0$ then $S(t \mid u)=g(t, \infty \mid u ; S(\cdot \mid \cdot)) / f_{V}(t)$ is also uniquely determined by $g$. Moreover, if $t_{o} \in \mathcal{S}_{F_{V}} \cup \mathcal{S}_{F_{U}}, \exists$ a sequence of $t_{j} \rightarrow t_{o}$ and $f_{U}\left(t_{j}\right)>0$ or $f_{V}\left(t_{j}\right)>0$, then $S\left(t_{o} \mid u\right)$ is also uniquely determined by $g$, as the $S(t \mid u)$ is continuous and $S\left(t_{j} \mid u\right)$ is identifiable $\forall j$. Thus given $g(\cdot, \cdot \mid u ; S(\cdot \mid \cdot)), S(t \mid u)$ is identifiable at each point $t$ in $\mathcal{S}_{F_{U}} \cup \mathcal{S}_{F_{V}}$.

Lemma 2. The parameter $\beta$ is not identifiable if $\mathcal{S}_{F_{L}} \cup \mathcal{S}_{F_{R}}$ is finite.

Proof. It suffices to give a counterexample under the semi-parametric set-up. Consider the $\mathrm{PH}$ model $h(t \mid u)=\exp ((t-a) u \beta \mathbf{1}(t \geq a)) h_{o}(t)$, where $\beta=1, h_{o}(x)=1(x \geq 0)$ and $u \in\{0,1\}$. WLOG, we can assume Case 2 interval censoring, with the two follow-up times $U$ and $V$, and $(Y, u) \perp(U, V)$. Moreover, we can assume that the support set $\mathcal{S}_{F_{U}} \cup \mathcal{S}_{F_{V}}=\{1,2,3, \ldots, n\}$, where $a=1$. Then $S(t \mid u)$ is identifiable at $t \in\{1, \ldots, n\}$, where $u \in\{0,1\}$. Thus by Corollary $1, S_{o}(a), S_{o}(a) \exp \left(-\int_{1}^{j} h_{o}(x) d x\right)$ and $S_{o}(a) \exp \left(-\int_{1}^{j} e^{\beta(x-1)} h_{o}(x) d x\right)$ are identifiable, where $j=1, \ldots, n$. Consequently,

$$
\int_{j}^{j+1} h_{o}(x) d x=c_{2 j-1} \text { and } \int_{j}^{j+1} e^{\beta(x-1)} h_{o}(x) d x=c_{2 j}, j \in\{1, \ldots, n-1\},
$$

where $c_{2 j-1}=1$ and $c_{2 j}=e^{(j-1) \beta} \frac{e^{\beta}-1}{\beta}=e^{j-1}(e-1)$ are given, but $h_{o}$ and $\beta$ are parameters, though their true values are $h_{o}(x)=1(x \geq 0)$ and $\beta=1$.

It is obvious that $(1(x \geq 0), 1)$ is a solution to $\left(h_{o}, \beta\right)$. We shall show that there is another solution to $\left(h_{o}, \beta\right)$ (where $\left.\beta>1\right)$ to $(2.2)$. That is, for $j \in\{1, \ldots, n-1\}$, we shall define another function $h_{o}$, say $h_{2}(\cdot)$ on $(j, j+1]$ such that

$$
\int_{j}^{j+1} h_{2}(x) d x=c_{2 j-1}=1 \text { and } \int_{j}^{j+1} e^{\beta(x-1)} h_{2}(x) d x=c_{2 j}=e^{j-1}(e-1) .
$$

For each $\gamma>1$, setting $h_{o}=h_{2}=\gamma c_{2 j-1} \mathbf{1}(x \in(j, j+1 / \gamma])$, where $x \in(j, j+1]$, then (2.3) yields

$$
e^{\beta(j-1)} \frac{e^{\beta / \gamma}-1}{\beta / \gamma}=c_{2 j} / c_{2 j-1}=e^{j-1}(e-1), \gamma>1,
$$

which specifies a different solution for $\beta$, that is, $\beta \neq 1$,

In particular, for $j=1,(2.4)$ becomes $\frac{e^{\beta / \gamma}-1}{\beta / \gamma}=e-1, \gamma>1$. Its solution is $\beta=\gamma$. The range of the solution $\beta$ to $(2.3)$ for $\gamma \geq 1$ and $j=1$ is $B_{1}=[1, \infty)$. For $j \in\{2, \ldots, n-1\}$, letting $\gamma \rightarrow \infty,(2.4)$ yields $e^{\beta(j-1)}=e^{j-1}(e-1)$, or

$$
\beta=\frac{j-1+\ln (e-1)}{j-1}>1, j \in\{2, \ldots, n-1\} .
$$

Since for each $j \in\{2, \ldots, n-1\}$, the solution $\beta$ to $(2.4)$ is continuous in $\gamma \in[1, \infty)$, the range of the solution $\beta$ to $(2.3)$ for $\gamma \geq 1$ is $B_{j}=\left[1,1+\frac{\ln (e-1)}{j-1}\right)$. It is easy to show that the range $B_{j}$ is decreasing in $j$. Here, notice that for each $j$, we only need to modify $h_{o}$ or $h_{2}$ in the interval $(j, j+1]$.

Since $n$ is finite, $\cap_{j=1}^{n-1} B_{j}=\left[1,1+\frac{\ln (e-1)}{n-2}\right)$ and $\left(1,1+\frac{\ln (e-1)}{n-2}\right) \neq \emptyset, \exists \beta_{o} \in\left(1,1+\frac{\ln (e-1)}{n-2}\right)$ such that $\beta=\beta_{o}$ is a solution to $e^{\beta(j-2)} \frac{e^{\beta / \gamma_{j}}-1}{\beta / \gamma_{j}}=e^{j-1}(e-1)$ for some $\gamma_{j}$, where $\gamma_{j}$ depends on $j \in\{1,2, \ldots, n-1\}$. This is the second solution of $\beta$ to $(2.2)$. $\square$

Theorem 2. An identifiability condition for $\beta$ under the mixed case IC model is that $\mathcal{S}_{F_{L}} \cup \mathcal{S}_{F_{R}}$ contains infinitely many points $\left\{t_{j}\right\}_{j \geq 1}$ with a limiting point, say $t_{o}=\lim _{j \rightarrow \infty} t_{j}$ in $(a, \infty)$, provided that $S_{o}^{\prime}\left(t_{o}\right) \neq 0$.

Proof. If $t_{j} \in \mathcal{S}_{F_{L}} \cup \mathcal{S}_{F_{R}}, S\left(t_{j} \mid u\right)$ is identifiable for $u=0$ or 1 . Since $S_{o}$ and $S(t \mid 1)$ are continuous, and $t_{j} \rightarrow t_{o}, S_{o}\left(t_{o}\right)$ and $S\left(t_{o} \mid 1\right)$ are also identifiable, and it leads to that $\left(S_{o}^{\prime}\left(t_{o}\right), S^{\prime}\left(t_{o} \mid 1\right)\right)$ is identifiable, as $S_{o}^{\prime}\left(t_{o}\right)=\lim _{t_{j} \rightarrow t_{o}} \frac{S_{o}\left(t_{j}\right)-S_{o}\left(t_{o}\right)}{t_{j}-t_{o}}$, etc.. They further lead to that $\left(h_{o}\left(t_{o}\right), h\left(t_{o} \mid 1\right)\right)$ is identifiable, as $h\left(t_{o} \mid 1\right)=-\frac{S^{\prime}\left(t_{o} \mid 1\right)}{S\left(t_{o} \mid 1\right)}$ etc.. Consequently $\beta$ can be identified by $h\left(t_{o} \mid 1\right)=e^{\left(t_{o}-a\right) \beta} h_{o}\left(t_{o}\right)$, as $a$ is known. $\square$

In the proof of Lemma 2, since $\left(S_{o}(t), S(t \mid 1)\right)$ are only identifiable at finitely many points and one cannot derive $\left(S_{o}^{\prime}(t), S^{\prime}(t \mid 1)\right)$ and $\left(h_{o}(t), h(t \mid 1)\right)$ through these finitely many points. Moreover, in view of the proof of Theorem 2, if $\left(S_{o}(t), S(t \mid 1)\right)$ are only identifiable at all positive integers, or $S_{o}^{\prime}\left(t_{o}\right)=0$ for the $t_{o}$ defined in Theorem 2 , then $\beta$ is likely non-identifiable. Moreover, the interval $(a, \infty)$ in Theorem 2 cannot be replaced by $[a, \infty)$. 
Lemma 3. The function $S_{o}(a)$ is not identifiable if a $\notin \mathcal{S}_{F_{L}} \cup \mathcal{S}_{F_{R}}$, even if $\beta$ is known and $\mathcal{S}_{F_{L}} \cup \mathcal{S}_{F_{R}}$ contains a nonempty open interval in $[a, \infty)$.

Proof. It suffices to give a counterexample. Consider a Case $1 \mathrm{IC}$ model with the censoring variable $C \sim U(2,3)$ and $h(t \mid u)=e^{u(t-1) \beta \mathbf{1}(t \geq 1)} h_{o}(t), u \in\{0,1\}$. Then $\beta$ is identifiable by Theorem 2 . Let $\beta=1$. If $h_{o}=1(t>0)$, then $S_{o}(a)=e^{-1}, S_{o}(2)=e^{-2}$, and $S(2 \mid 1)=e^{-1} e^{-(e-1)}$. Let

$$
h_{0}^{*}(x)= \begin{cases}h_{1} & \text { if } x \in(0,1] \\ h_{2} & \text { if } x \in(1,1.5] \\ h_{3} & \text { if } x \in(1.5,2] \\ \text { arbitrary } & \text { otherwise. }\end{cases}
$$

The equation represents a hyperplane in $R^{3}$ passing through $(1,1,1)$. Thus there are many solutions near $(1,1,1)$ satisfying $\left(h_{1}, h_{2}, h_{3}\right) \geq 0$ and $h_{1} \neq 1$. Thus the solution to $S_{o}(a)=e^{-h_{1}}$ is not unique. $\square$

Remark 1. It is interesting to notice the following facts in the univariate covariate case.

1. If the covariate is time-independent then $\beta$ is identifiable even if $\mathcal{S}_{F_{L}} \cup \mathcal{S}_{F_{R}}$ contains only one point $t$ at which $S(t) \in(0,1)$. In fact, if $t_{o} \in \mathcal{S}_{F_{L}} \cup \mathcal{S}_{F_{R}}$ then $S\left(t_{o} \mid u\right)=\left(S_{o}\left(t_{o}\right)\right)^{e^{\beta u}}$ is identifiable for $u \in\{0,1\}$ and thus $S\left(t_{o} \mid 0\right)=S_{o}\left(t_{o}\right)$ is identifiable. Since $\beta=\ln \frac{\ln S\left(t_{o} \mid 1\right)}{\ln S_{o}\left(t_{o}\right)}$ and both $S_{o}\left(t_{o}\right)$ and $S\left(t_{o} \mid 1\right)$ are known, it follows that $\beta$ is identifiable.

2. If the covariate is $z=u \mathbf{1}(t \geq a)$, then $\beta$ and $S_{o}(a)$ are identifiable even if $\left(\mathcal{S}_{F_{L}} \cup \mathcal{S}_{F_{R}}\right) \cap[a, \infty)$ contains only two points $t$ 's at which $S(t) \in(0,1)$. In fact, let $a \leq b<c$, where $b, c \in \mathcal{S}_{F_{L}} \cup \mathcal{S}_{F_{R}}$ and $1>S_{o}(a) \geq S_{o}(b) \geq S_{o}(c)>0$, then $\left(S_{o}(b), S_{o}(c), S(b \mid 1), S(c \mid 1)\right)$ is identifiable. Moreover, $S(t \mid 1)=\left(S_{o}(a)\right)^{1-e^{\beta}}\left(S_{o}(t)\right)^{e^{\beta}}$ if $t>a$. Since $\frac{S(b \mid 1)}{S(c \mid 1)}=\left(\frac{S_{o}(b)}{S_{o}(c)}\right)^{e^{\beta}}, \beta$ is identifiable. Since $S(b \mid 1)=$ $\left(S_{o}(a)\right)^{1-e^{\beta}}\left(S_{o}(b)\right)^{e^{\beta}}, S_{o}(a)$ is also identifiable.

3. However, if $z=(t-a) u \mathbf{1}(t \geq a)$, then neither $\beta$ nor $S_{o}(a)$ is identifiable even if the set $\left(\mathcal{S}_{F_{L}} \cup \mathcal{S}_{F_{R}}\right) \cap[a, \infty)$ contains countably many points in $(a, \infty)$ (see the comment after Theorem 2). Moreover, $S_{o}(a)$ is not identifiable even if $\beta$ is known and $\mathcal{S}_{F_{L}} \cup \mathcal{S}_{F_{R}}$ contains a non-empty open interval in $[a, \infty)$.

These facts imply that if the random vector $(L, R)$ only takes on finitely many values, there exist consistent estimators of $\beta$ under the $\mathrm{PH}$ model with time-independent covariates but not under the situation considered in this paper. Also, in simulation studies, one can let the censoring vector have a finite discrete distribution under the $\mathrm{PH}$ model with time-independent covariates, but should not try the censoring vector which takes on finitely many values if the covariate is given by (1.2).

\section{Semi-parametric Estimation}

We shall propose our estimation method in this section.

\subsection{Preliminary}

For the PH model with time-independent covariates, the generalized likelihood function with IC data is given by $\mathcal{L}_{*}=\prod_{i=1}^{n} \mu_{S(\cdot \cdot)}\left(I_{i}\right)$ as in (2.1). For the time-dependent covariates $\mathbf{z}(t)=\mathbf{u} \cdot(t-a) \mathbf{1}(t \geq a)$, such a definition would lead to $\mathcal{L}_{*}=0$ in view of Theorem 1 , as $\mu_{S(\cdot \cdot))}\left(I_{i}\right)=0$ if $L_{i}=R_{i}$. The first modification to $\mathcal{L}_{*}$ is

$$
\mathcal{L}_{o}=\prod_{i=1}^{n} \mu_{S(\cdot \mid \cdot)}\left(I_{i}^{*}\right), \text { where } \mu_{S}\left(I_{i}^{*}\right)=S\left(L_{i}^{*}\right)-S\left(R_{i}\right), \quad L_{i}^{*}= \begin{cases}L_{i} & \text { if } L_{i}<R_{i} \\ L_{i}-\epsilon_{n} & \text { if } L_{i}=R_{i}\end{cases}
$$

and $\epsilon_{n}=\frac{1}{n} \wedge \min \left\{|x-y|: x \neq y, x, y \in\left\{a, L_{1}, \ldots, L_{n}, R_{1}, \ldots, R_{n}\right\}\right\}$. Remark 2. Hereafter, by abuse of notations, we write $L_{i}=L_{i}^{*}, i=1, \ldots, n$. Moreover, $I_{i}$ 's are the modified observed intervals, instead of the original ones, that is, $I_{i}=\left(L_{i}^{*}, R_{i}\right]$.

An interval $A$ is called an innermost interval (II) if it is an intersection of the observed intervals $I_{i}$ 's and if $A \cap I_{i}=A$ or $\emptyset$ for each $I_{i}$. Notice that each exact observation $\left[Y_{k}, Y_{k}\right]\left(=\left[L_{k}, R_{k}\right]\right)$ is an II, however, under our modification, it is changed to an interval $\left(Y_{k}-\epsilon_{n}, Y_{k}\right]$. It is well known (see Wong 
and $\mathrm{Yu}(2017))$ that under the $\mathrm{PH}$ model with time-independent covariates in order to maximize $\mathcal{L}_{o}$, it suffices to put the weights of $S_{o}$ to the IIs. Moreover, the weight to each II is uniquely determined, but not the distribution of the weight within the II. Let $A_{1}, \ldots, A_{m}$ be all the II's induced by $I_{i}$ 's and let $\left(v_{j}, w_{j}\right)$ be the pair of endpoints of $A_{j}$, where $w_{0}=-\infty<w_{1}<w_{2}<\cdots<w_{m} \leq \infty$. For each $i$, let $\xi_{i}=\mathbf{1}\left(R_{i}<w_{m}\right)$ and define $l_{i}$ and $r_{i}$ by $w_{r_{i}} \leq R_{i}<w_{r_{i}+1}$ and $w_{l_{i}} \leq L_{i}<w_{l_{i}+1}$. Then the likelihood function in (2.1) becomes

$$
\begin{aligned}
& \mathcal{L}_{o}\left(\beta, S_{o}\right)=\prod_{R_{i} \leq a \text { or } \mathbf{u}_{i}=0}\left(S_{o}\left(w_{l_{i}}\right)-S_{o}\left(w_{r_{i}}\right)\right) \\
& \cdot \prod_{L_{i}<a<R_{i}, \mathbf{u}_{i} \neq 0}\left\{S_{o}\left(w_{l_{i}}\right)-\xi_{i} S_{o}(a) \exp \left(-\sum_{w_{j} \in\left[a, R_{i}\right]} \int_{v_{j}}^{w_{j}} e^{\beta(x-a)} h_{o}(x) d x\right)\right\} \cdot \prod_{L_{i}>a, \mathbf{u}_{i} \neq 0} S_{o}(a) \\
& \cdot\left[\exp \left(-\sum_{w_{j} \in\left(a, L_{i}\right]} \int_{v_{j}}^{w_{j}} e^{\beta(x-a)} h_{o}(x) d x\right)-\xi_{i} \exp \left(-\sum_{w_{j} \in\left(a, R_{i}\right]} \int_{v_{j}}^{w_{j}} e^{\beta(x-a)} h_{o}(x) d x\right)\right] .
\end{aligned}
$$

However, there is still a problem in this definition, as $h_{o}$ is a function of $x$ and needs to be properly defined on $\left[v_{j}, w_{j}\right]$ for all $j<m$ (note that $\left.S_{o}\left(w_{m}\right)=0\right)$. The counterexample constructed in the proof of Lemma 2 can actually be modified to show that the definition of $h_{o}$ on the IIs can change the value of the "SMLE" of $\beta$. There are two naive approaches:

A1. Let $h_{o}$ be constant at each $A_{1}, \ldots, A_{m-1}$.

A2. Let $h_{o}$ be two-piecewise-constant at each $A_{1}, \ldots, A_{m-1}$.

We shall explain in $\S 3.3$ that the previous two naive approaches do not lead to a consistent SMLE. We propose the third approach as follows, in addition to the modification mentioned in Remark 2 about $L_{i}$ 's and $I_{i}$ 's.

A3. First, let $h_{o}(x)=0$ if $x \notin \cup_{k}\left(v_{k}, w_{k}\right]$, where $\left(v_{1}, w_{1}\right], \ldots,\left(v_{m}, w_{m}\right]$ are all the II's. Moreover, notice that each $\left(v_{k}, w_{k}\right]$ will be contained by several modified observed intervals $I_{i}$ 's (see Remark 2) with $J$ $(\geq 1)$ distinct values of $\mathbf{u}_{i}$ 's, where $J$ depends on $k$. There are two types of $\left(v_{k}, w_{k}\right):(1) w_{k}-v_{k} \approx 0$, or $w_{k} \leq a$, or $w_{k}-a \approx 0$ and $a \in\left(v_{k}, w_{k}\right]$; (2) otherwise. For $k<m$, define

$h_{o}(x)=\left\{\begin{array}{ll}\text { constant on }\left(v_{k}, w_{k}\right] & \text { if }\left(v_{k}, w_{k}\right) \text { belongs to type }(1) \\ J \text {-piece-wise constant on }\left(v_{k}, w_{k}\right] & \text { if }\left(v_{k}, w_{k}\right) \text { belongs to type (2) }\end{array}\right.$ (in particular, if $\left(v_{k}, w_{k}\right)$ belongs to type (2), then

$$
h_{o}(x)=\sum_{j=1}^{J} h_{k j} \mathbf{1}\left(x \in\left(v_{k j}, w_{k j}\right]\right) \text { for } x \in\left(v_{k}, w_{k}\right], \text { where } v_{k}=v_{k 1}, w_{k 1}=v_{k 2}
$$

$w_{k 2}=v_{k 3}, \ldots, w_{k J}=w_{k}$ and $w_{k j}-v_{k j}=\left\{\begin{array}{ll}\frac{w_{k}-v_{k}}{J} & \text { if } a \in\left(v_{k}, w_{k}\right], j \in\{1, \ldots, J\} \\ \frac{w_{k}-v_{k}}{J-1} & \text { if } a \notin\left(v_{k}, w_{k}\right], j \in\{2, \ldots, J\}\end{array}\right)$. If $k=m$, simply define $S_{o}\left(w_{m}\right)=0\left(h_{o}\right.$ can be arbitrary on $\left(v_{m}, w_{m}\right]$, provided that $h_{o} \geq 0$ and $\left.\int_{v_{m}}^{w_{m}} h_{o}(x) d x=\infty\right)$.

Remark 3. By abuse of notations, we let $\left(a_{j}, b_{j}\right]$ be the interval in which $h_{o}$ is constant, as specified in (3.3). Then

$$
h_{o}(x)=\sum_{j} h_{j} \mathbf{1}\left(x \in\left(a_{j}, b_{j}\right]\right) \text {, where }\left(a_{j}, b_{j}\right] \text { may not be an II. }
$$


In view of $(3.4), \mathcal{L}_{o}$ in $(3.2)$ becomes

$$
\begin{aligned}
\mathcal{L}_{o}\left(\beta, S_{o}\right)= & \prod_{R_{i} \leq a, \text { or } \mathbf{u}_{i}=0}\left\{\exp \left(-\sum_{b_{j} \leq L_{i}} h_{j}\left[b_{j}-a_{j}\right]\right)\left[1-\exp \left(-\sum_{b_{j} \in\left(L_{i}, R_{i}\right]} h_{j}\left[b_{j}-a_{j}\right]\right)\right]^{\xi_{i}}\right\} \\
& \cdot \prod_{L_{i} \geq a, \mathbf{u}_{i} \neq 0}\left\{S_{o}(a) \exp \left(-\frac{e^{-a \mathbf{u}_{i} \beta}}{\mathbf{u}_{i} \beta} \sum_{b_{j} \in\left(a, L_{i}\right]} h_{j}\left[e^{\mathbf{u}_{i} \beta b_{j}}-e^{\mathbf{u}_{i} \beta a_{j}}\right]\right)\right. \\
& \left.\cdot\left[1-\exp \left(-\frac{e^{-a \mathbf{u}_{i} \beta}}{\mathbf{u}_{i} \beta} \sum_{b_{j} \in\left(L_{i}, R_{i}\right]} h_{j}\left[e^{\mathbf{u}_{i} \beta b_{j}}-e^{\mathbf{u}_{i} \beta a_{j}}\right]\right)\right]\right\} \\
& \cdot \prod_{L_{i}<a<R_{i}, \mathbf{u}_{i} \neq 0}\left\{\exp \left(-\sum_{b_{j} \leq L_{i}} h_{j}\left[b_{j}-a_{j}\right] \xi_{i}\right)\right. \\
& \left.\left.\cdot\left[1-\exp \left(-\sum_{b_{j} \in\left(L_{i}, a\right]} h_{j}\left(b_{j}-a_{j}\right)-\frac{e^{-a \mathbf{u}_{i} \beta}}{\mathbf{u}_{i} \beta} \sum_{b_{j} \in\left(L_{i}, R_{i}\right]} h_{j}\left[e^{\mathbf{u}_{i} \beta b_{j}}-e^{\mathbf{u}_{i} \beta a_{j}}\right]\right)\right]\right]^{\xi_{i}}\right\} .
\end{aligned}
$$

\subsection{Definition of the Modified Likelihood Function}

As explained later in (3.11), it is more convenient to replace $h_{o}(x) e^{\beta \mathbf{u}_{i} x}$ by a piecewise constant function, and to modify $\mathcal{L}_{o}$ as

$$
\begin{aligned}
& \mathcal{L}=\prod_{R_{i} \leq a, \text { or } \mathbf{u}_{i}=0}\left\{\exp \left(-\sum_{b_{j} \leq L_{i}} h_{j}\left[b_{j}-a_{j}\right]\right)\left[1-\exp \left(-\sum_{b_{j} \in\left(L_{i}, R_{i}\right]} h_{j}\left[b_{j}-a_{j}\right]\right)\right]^{\xi_{i}}\right\} \\
& \cdot \prod_{L_{i} \geq a, \mathbf{u}_{i} \neq 0}\left\{\exp \left(-\sum_{b_{j} \leq a}\left(b_{j}-a_{j}\right) h_{j}-\sum_{b_{j} \in\left(a, L_{i}\right]} h_{j} e^{\left(b_{j}-a\right) \mathbf{u}_{i} \beta}\left(b_{j}-a_{j}\right)\right)\right. \\
& \left.\cdot\left[1-\exp \left(-\sum_{b_{j} \in\left(L_{i}, R_{i}\right]} h_{j} e^{\mathbf{u}_{i} \beta\left(b_{j}-a\right)}\left(b_{j}-a_{j}\right)\right)\right]^{\xi_{i}}\right\} \prod_{L_{i}<a<R_{i}, \mathbf{u}_{i} \neq 0}\left\{\exp \left(-\sum_{b_{j} \leq L_{i}} h_{j}\left[b_{j}-a_{j}\right]\right)\right. \\
& \left.\left[1-\exp \left(-\sum_{b_{j} \in\left(L_{i}, a\right]} h_{j}\left[b_{j}-a_{j}\right]-\sum_{b_{j} \in\left(a, R_{i}\right]} h_{j} e^{\left(b_{j}-a\right) \mathbf{u}_{i} \beta}\left[b_{j}-a_{j}\right]\right)\right]^{\xi_{i}}\right\}, \\
& \ln \mathcal{L}=\sum_{R_{i} \leq a, \text { or }}\left\{\left(-\sum_{b_{j} \leq L_{i}} h_{j}\left[b_{j}-a_{j}\right]\right)+\xi_{i} \ln \left[1-\mathcal{U}_{i}\right]\right\} \\
& +\sum_{L_{i} \geq a, \mathbf{u}_{i} \neq 0}\left\{-\sum_{b_{j} \leq a} h_{j}\left(b_{j}-a_{j}\right)-\sum_{b_{j} \in\left(a, L_{i}\right]} h_{j} e^{\left(b_{j}-a\right) \mathbf{u}_{i} \beta}\left(b_{j}-a_{j}\right)+\xi_{i} \ln \left[1-\mathcal{W}_{i}\right]\right\} \\
& \left.+\sum_{L_{i}<a<R_{i}, \mathbf{u}_{i} \neq 0}\left\{-\sum_{b_{j} \leq L_{i}} h_{j}\left[b_{j}-a_{j}\right]+\xi_{i} \ln \left[1-\mathcal{V}_{i}\right]\right]\right\}, \\
& \text { where } \mathcal{U}_{i}=\exp \left(-\sum_{b_{j} \in\left(L_{i}, R_{i}\right]} h_{j}\left[b_{j}-a_{j}\right]\right), \mathcal{W}_{i}=\exp \left(-\sum_{b_{j} \in\left(L_{i}, R_{i}\right]} h_{j} e^{\mathbf{u}_{i} \beta\left(b_{j}-a\right)}\left(b_{j}-a_{j}\right)\right) \text {, } \\
& \text { and } \mathcal{V}_{i}=\exp \left(-\sum_{b_{j} \in\left(L_{i}, a\right]} h_{j}\left[b_{j}-a_{j}\right]-\sum_{b_{j} \in\left(a, R_{i}\right]} h_{j} e^{\left(b_{j}-a\right) \mathbf{u}_{i} \beta}\left[b_{j}-a_{j}\right]\right) \text {. }
\end{aligned}
$$

The SMLE maximizes $\ln \mathcal{L}$ over all $h_{j}$ 's and $\beta$. It is well known that the Newton Raphson method does not work (see Wong and Yu (2012) or Appendix II). We suggest to use the steepest decent method. Thus 
we derive the derivatives as follows.

$$
\begin{aligned}
& \frac{\partial \ln \mathcal{L}}{\partial \beta}=\sum_{L_{i} \geq a, \mathbf{u}_{i} \neq 0}\left\{-\sum_{b_{j} \in\left(a, L_{i}\right]} H_{i j}+\frac{\xi_{i} \mathcal{W}_{i}}{\left[1-\mathcal{W}_{i}\right]} \sum_{b_{j} \in\left(L_{i}, R_{i}\right]} H_{i j}\right\}+\sum_{L_{i}<a<R_{i}, \mathbf{u}_{i} \neq 0} \frac{\xi_{i} \mathcal{V}_{i}}{1-\mathcal{V}_{i}} \sum_{b_{j} \in\left(a, R_{i}\right]} H_{i j} \\
& \left(H_{i j}=h_{j} e^{\left(b_{j}-a\right) \mathbf{u}_{i} \beta}\left(b_{j}-a_{j}\right)\left(b_{j}-a\right) \mathbf{u}_{i}\right) \\
& =-\sum_{L_{i} \geq a, \mathbf{u}_{i} \neq 0}\left\{\sum_{b_{j} \in\left(a, L_{i}\right]} H_{i j}+\xi_{i}\left(1-\frac{1}{1-\mathcal{W}_{i}}\right) \sum_{b_{j} \in\left(L_{i}, R_{i}\right]} H_{i j}\right\} \\
& -\sum_{L_{i}<a<R_{i}, \mathbf{u}_{i} \neq 0} \xi_{i}\left(1-\frac{1}{1-\mathcal{V}_{i}}\right) \sum_{b_{j} \in\left(a, R_{i}\right]} H_{i j} \\
& \frac{\partial \ln \mathcal{L}}{\partial h_{k}}=-\left(b_{k}-a_{k}\right) \cdot\left\{\sum_{R_{i} \leq a, \text { or } \mathbf{u}_{i}=0}\left\{\mathbf{1}\left(b_{k} \leq L_{i}\right)-\mathbf{1}\left(b_{k} \in\left(L_{i}, R_{i}\right]\right) \frac{\xi_{i} \mathcal{U}_{i}}{1-\mathcal{U}_{i}}\right\}\right. \\
& +\sum_{L_{i} \geq a, \mathbf{u}_{i} \neq 0}\left\{\mathbf{1}\left(b_{k} \leq a\right)+\mathbf{1}\left(b_{k} \in\left(a, L_{i}\right]\right) e^{\left(b_{k}-a\right) u_{k} \beta}-\xi_{i} \mathbf{1}\left(b_{k} \in\left(L_{i}, R_{i}\right]\right) e^{u_{k} \beta\left(b_{k}-a\right)} \frac{\mathcal{W}_{i}}{1-\mathcal{W}_{i}}\right\} \\
& \left.+\sum_{L_{i}<a<R_{i}, \mathbf{u}_{i} \neq 0}\left\{\mathbf{1}\left(b_{k} \leq L_{i}\right)-\xi_{i}\left[\mathbf{1}\left(b_{k} \in\left(L_{i}, a\right]\right)+\mathbf{1}\left(b_{k} \in\left(a, R_{i}\right]\right) e^{\left(b_{k}-a\right) u_{k} \beta}\right] \frac{\mathcal{V}_{i}}{1-\mathcal{V}_{i}}\right\}\right\} \\
& =-\left(b_{k}-a_{k}\right) \cdot\left\{\sum _ { R _ { i } \leq a , \text { or } } \left\{\mathbf{\mathbf { u } _ { i } = 0}\left\{\left(b_{k} \leq L_{i}\right)+\xi_{i} \mathbf{1}\left(b_{k} \in\left(L_{i}, R_{i}\right]\right)\left(1-\frac{1}{1-\mathcal{U}_{i}}\right)\right\}\right.\right. \\
& +\sum_{L_{i} \geq a, \mathbf{u}_{i} \neq 0}\left\{\mathbf{1}\left(b_{k} \leq a\right)+e^{\left(b_{k}-a\right) u_{k} \beta}\left[\mathbf{1}\left(b_{k} \in\left(a, L_{i}\right]\right)+\xi_{i} \mathbf{1}\left(b_{k} \in\left(L_{i}, R_{i}\right]\right)\left(1-\frac{1}{1-\mathcal{W}_{i}}\right)\right]\right\} \\
& \left.+\sum_{L_{i}<a<R_{i}, \mathbf{u}_{i} \neq 0}\left\{\mathbf{1}\left(b_{k} \leq L_{i}\right)+\xi_{i}\left[\mathbf{1}\left(b_{k} \in\left(L_{i}, a\right]\right)+\mathbf{1}\left(b_{k} \in\left(a, R_{i}\right]\right) e^{\left(b_{k}-a\right) u_{k} \beta}\right]\left(1-\frac{1}{1-\mathcal{V}_{i}}\right\}\right)\right\} .
\end{aligned}
$$

To estimate the covariance matrix of $\hat{\beta}$, we need to compute

$$
\begin{aligned}
& \frac{\partial^{2} \ln \mathcal{L}}{\partial \beta^{2}}=-\sum_{L_{i}<a<R_{i}, \mathbf{u}_{i} \neq 0} \xi_{i}\left\{\sum_{b_{j} \in\left(a, R_{i}\right]} H_{i j}^{\prime}-\frac{\sum_{b_{j} \in\left(a, R_{i}\right]} H_{i j}^{\prime}}{1-\mathcal{V}_{i}}+\frac{\mathcal{V}_{i}\left(\sum_{b_{j} \in\left(a, R_{i}\right]} H_{i j}\right)^{2}}{\left(1-\mathcal{V}_{i}\right)^{2}}\right\} \\
& -\sum_{i: L_{i} \geq a, \mathbf{u}_{i} \neq 0}\left\{\sum_{b_{j} \in\left(a, L_{i}\right]} H_{i j}^{\prime}+\xi_{i}\left[\sum_{b_{j} \in\left(L_{i}, R_{i}\right]} H_{i j}^{\prime}-\frac{\sum_{b_{j} \in\left(L_{i}, R_{i}\right]} H_{i j}^{\prime}}{1-\mathcal{W}_{i}}+\frac{\mathcal{W}_{i}\left(\sum_{b_{j} \in\left(L_{i}, R_{i}\right]} H_{i j}\right)^{2}}{\left(1-\mathcal{W}_{i}\right)^{2}}\right]\right\} \\
& \text { ( where } \left.H_{i j}^{\prime}=h_{j} e^{\left(b_{j}-a\right) \mathbf{u}_{i} \beta}\left(b_{j}-a_{j}\right)\left(\left(b_{j}-a\right) \mathbf{u}_{i}\right)^{2}\right) \text {. } \\
& \frac{\partial^{2} \ln \mathcal{L}}{\partial h_{k}^{2}}=-\left(b_{k}-a_{k}\right)^{2}\left\{\sum_{R_{i} \leq a \text { or } \mathbf{u}_{i}=0} \xi_{i} \mathbf{1}\left(b_{k} \in\left(L_{i}, R_{i}\right]\right) \frac{\mathcal{U}_{i}}{\left(1-\mathcal{U}_{i}\right)^{2}}\right. \\
& +\sum_{L_{i} \geq a, \mathbf{u}_{i} \neq 0} \xi_{i} \mathbf{1}\left(b_{k} \in\left(L_{i}, R_{i}\right]\right) \frac{e^{2\left(b_{k}-a\right) u_{k} \beta} \mathcal{W}_{i}}{\left(1-\mathcal{W}_{i}\right)^{2}} \\
& \left.+\sum_{L_{i}<a<R_{i}, \mathbf{u}_{i} \neq 0} \xi_{i}\left[\mathbf{1}\left(b_{k} \in\left(L_{i}, a\right]\right)+\mathbf{1}\left(b_{k} \in\left(a, R_{i}\right]\right) e^{2\left(b_{k}-a\right) u_{k} \beta}\right] \frac{\mathcal{V}_{i}}{\left(1-\mathcal{V}_{i}\right)^{2}}\right\} \text {. }
\end{aligned}
$$

Remark 4. As explained later in Example 3.1, the semi-parametric approach specified in (3.3) essentially estimates $\mu_{F}(I I)$ by the GMLE with given $\beta$ if the length of the II is not so small. There are cases that the length of an II may not tend to 0 such as an II containing [0,20] in a mammogram data set, as the age for the first mammogram is likely to be larger than 20 years. It is a practical issue how to interpret an II being small. 


\subsection{Justification of $\mathcal{L}_{o}$ and $\mathcal{L}$}

We shall also explain why the third modifications of the likelihood works through Example 3.1, as well as why the others do not work.

Example 3.1. Consider a continuous nonnegative random variable $Y$ that satisfies the model $h(t \mid u)=$ $h_{o}(t) e^{t u \beta \mathbf{1}(t \geq 0)}$, where $u \sim \operatorname{bin}(m, 0.5)$, the binomial distribution with $m$ trials and the probability of success $p=0.5, h_{o}$ is continuous at $c=0.69$ and $h_{o}(c) \neq 0$. Let $Y$ be subject to a Case 2 IC model, where the censoring vector $(U, V) \equiv\left(c, c+c_{n}\right), c_{n}=4 n^{-1 / 2}$ and $n$ is the sample size. Then the observations are of the forms $(0, c, u),\left(c, c+c_{n}, u\right)$, or $\left(c+c_{n}, \infty, u\right), u \in\{0,1, \ldots, m\}$. The observed intervals are either $(0, c],\left(c, c+c_{n}\right]$, or $\left(c+c_{n}, \infty\right)$, and they are all IIs. $J$ defined in A3 satisfies $j=m+1$ if $n$ is large enough. One may think that $\beta$ is not identifiable as the support set is finite, in view of Lemma 2. Notice that $(U, V)$ is really $\left(U_{n}, V_{n}\right)$ and one can treat the support set being $A=\cup_{n}\left(\mathcal{S}_{F_{U_{n}}} \cup \mathcal{S}_{F_{V_{n}}}\right)$, which is not finite and has a limiting point $c>a=0$. For a fixed $n, S(t \mid u)$ is identifiable at $c$ and $c+c_{n}$. We shall first let $m=1$, i.e., $u \sim \operatorname{bin}(1,0.5)$. Then

$$
\begin{gathered}
\ln (S(t \mid u))=-\int_{0}^{t} h_{o}(x) e^{\beta u x} d x, u \in\{0,1\}, \ln \left(S(c \mid u) / S\left(c+c_{n} \mid u\right)\right)=\int_{c}^{c+c_{n}} h_{o}(x) e^{\beta u x} d x, u \in\{0,1\} \\
\lim _{n \rightarrow \infty} \frac{1}{c_{n}} \ln \left(S(c \mid u) / S\left(c+c_{n} \mid u\right)\right)=h_{o}(c) e^{\beta u c}, u \in\{0,1\}, \text { and } \frac{1}{c} \ln \frac{h_{o}(c) e^{1 \beta c}}{h_{o}(c) e^{0 \beta c}}=\beta
\end{gathered}
$$

Thus $\beta$ is identifiable. Therefore, it is possible to construct consistent estimators of $\beta$. We shall discuss four estimators as follows.

a. A GMLE approach. If $n$ is large enough, there are three innermost intervals $(0, c],\left(c, c+c_{n}\right]$ and $\left(c+c_{n}, \infty\right)$. Let $n_{1 u}, n_{2 u}$ and $n_{3 u}$ be the numbers of the three types of intervals with $u=0$ or 1 . Recall that the generalized likelihood function is $\mathcal{L}_{o}=(1-S(c \mid 0))^{n_{10}}(1-S(c \mid 1))^{n_{11}}\left(S(c \mid 0)-S\left(c+c_{n} \mid 0\right)\right)^{n_{20}}(S(c \mid 1)-$ $\left.S\left(c+c_{n} \mid 1\right)\right)^{n_{21}}\left(S\left(c+c_{n} \mid 0\right)\right)^{n_{30}}\left(S\left(c+c_{n} \mid 1\right)\right)^{n_{31}}$ and the GMLE of $S(\cdot \mid u)$, say $\hat{S}(\cdot \mid u)$, is given by

$$
\hat{S}(c \mid u)=\frac{n_{2 u}+n_{3 u}}{n_{\cdot u}} \text { and } \hat{S}\left(c+c_{n} \mid u\right)=\frac{n_{3 u}}{n_{\cdot u}}, u \in\{0,1\}, \text { where } u \cdot u=\sum_{j=1}^{3} n_{j u} .
$$

Replacing $S(\cdot \mid u)$ in the last two equations in $(3.5)$ by $\hat{S}(\cdot \mid u)$ yields an estimator of $\beta$ :

$$
\hat{\beta}=\frac{1}{c} \ln \frac{\ln \left(\hat{S}(c \mid 1) / \hat{S}\left(c+c_{n} \mid 1\right)\right)}{\ln \left(\hat{S}(c \mid 0) / \hat{S}\left(c+c_{n} \mid 0\right)\right)}=\frac{1}{c} \ln \frac{\frac{n_{21}}{n_{\cdot 1}-n_{21}}}{\frac{n_{20}}{n \cdot 0-n_{20}}} .
$$

Since it makes use of the GMLE $\hat{S}(\cdot \mid u)$, we can say that $\hat{\beta}$ is a GMLE of $\beta$. Simulation results in $\S 4$ suggest that the GMLE $\hat{\beta}$ is consistent (see Table 1 ).

b. An estimator due to the first naive approach. Let $h_{o}$ be constant in each of first two IIs, say it equals $h_{1}$ and $h_{2}$, respectively (i.e., $h_{o}(x)=h_{1} \mathbf{1}(x \in(0, c])+h_{2} \mathbf{1}\left(x \in\left(c, c+c_{n}\right]\right)$ if $x \in\left(0, c+c_{n}\right]$ and arbitrary otherwise). Then $\mathcal{L}_{o}$ in (3.1) satisfies $\mathcal{L}_{o} \approx \mathcal{L}_{1}$, where

$$
\begin{aligned}
\mathcal{L}_{1}= & \left(1-\exp \left(-c h_{1}\right)\right)^{n_{10}}\left[\exp \left(-c h_{1}\right)\left(1-\exp \left(-c_{n} h_{2}\right)\right)\right]^{n_{20}}\left(\exp \left(-\left(c h_{1}+c_{n} h_{2}\right)\right)\right)^{n_{30}} \\
& \times\left(1-\exp \left(-h_{1} \frac{e^{c \beta}-1}{\beta}\right)\right)^{n_{11}}\left[\exp \left(-h_{1} \frac{e^{c \beta}-1}{\beta}\right)\left(1-\exp \left(-h_{2} c_{n} e^{c \beta}\right)\right)\right]^{n_{21}} \quad\left(\text { as } c_{n} \approx 0\right) \\
& \times\left(\exp \left(-h_{1} \frac{e^{c \beta}-1}{\beta}-h_{2} c_{n} e^{c \beta}\right)\right)^{n_{31}} .
\end{aligned}
$$

Let $\check{\beta}$ be the value of $\beta$ that maximizes $\mathcal{L}_{1}$ just defined. Simulation results in Table 1 of Section 4 suggest that the estimator $\breve{\beta}$ is not consistent. Thus this approach does not work. In fact the limiting points of $\breve{\beta}$ should maximize the almost sure limit

$$
\begin{aligned}
\lim _{n \rightarrow \infty} \frac{\ln \mathcal{L}_{1}\left(h_{1}, h_{2}\right)}{n} & =p_{10} \ln \left(1-\exp \left(-c h_{1}\right)\right)+p_{30} \ln \exp \left(-\left(c h_{1}\right)\right) \quad\left(\text { note } c_{n} \rightarrow 0\right) \\
& +p_{11} \ln \left(1-\exp \left(-h_{1} \frac{e^{c \beta}-1}{\beta}\right)\right)+p_{31} \ln \left(\exp \left(-h_{1} \frac{e^{c \beta}-1}{\beta}\right)\right),
\end{aligned}
$$


where $p_{i u}$ 's are defined in an obvious way, e.g., $p_{1 u}=P(Y \leq c \mid u)$. Thus the (almost sure) limiting points of the estimator of $\left(h_{1}, h_{2}, \beta\right)$ should satisfy

$$
\begin{aligned}
& p_{30}=\exp \left(-\int_{0}^{c} h_{o}(x) d x\right)=\exp \left(-c h_{1}\right) \\
& p_{31}=\exp \left(-\int_{0}^{c} h_{o}(x) e^{\beta x} d x\right)=\exp \left(-h_{1} \frac{e^{c \beta}-1}{\beta}\right),
\end{aligned}
$$

etc.. If $\check{\beta}$ is consistent, then for each $\left(h_{o}(\cdot), \beta\right)$, there exists $h_{1}$ satisfying the two equations related to $p_{30}$ and $p_{31}$. Let $\beta=1$ and $h_{o}(x)=\mathbf{1}(x \notin(c / 2, c))$, then $p_{30}$ yields $h_{1}=0.5$ and $p_{31}$ yields $\exp \left(-\left(e^{c / 2}-1\right)\right)=\exp \left(-0.5\left(e^{c}-1\right)\right)$, a contradiction, as $c=0.69$. Thus the first naive approach does not lead to a consistent estimator of $\beta$.

Moreover, if $u \sim \operatorname{bin}(m, 0.5)$ with $m \geq 2$ in Example 3.1, then the second naive approach does not lead to a consistent estimator of $\beta$ neither. The argument is similar to the last paragraph. Here $J=m+1>2$, where $J$ is defined in A3, the example motivates Eq. (3.3).

c. A consistent estimator due to the second naive approach when $u \sim \operatorname{bin}(1,0.5)$. Define $h_{o}(x)=\left\{\begin{array}{ll}h_{1} & \text { if } x \in(0, c / 2] \\ h_{2} & \text { if } x \in(c / 2, c] \\ h_{3} & \text { if } x \in\left(c, c+c_{n} / 2\right] \\ h_{4} & \left.\text { if } x \in\left(c+c_{n} / 2, c+c_{n}\right]\right) \\ \text { arbitrary } & \text { if } x>c+c_{n} .\end{array}\right.$ There are 5 parameters in this approach and it can be

shown that the solution to $\left(\beta, h_{1}, h_{2}, h_{3}, h_{4}\right)$ that maximizes $\mathcal{L}_{o}$ is not unique. In fact let $\hat{S}(\cdot \mid u)$ be the GMLE given in (3.6) (which is uniquely defined only at $0, c$ and $\left.c+c_{n}\right)$, then $\forall \beta \in(-\infty, \infty)$, there is a unique solution of $\left(h_{1}, h_{2}, h_{3}, h_{4}\right)$ to the system of four linear equations in (3.8) and (3.9):

$$
\begin{gathered}
-\ln (\hat{S}(c \mid u))=h_{1} \int_{0}^{c / 2} e^{\beta u x} d x+h_{2} \int_{c / 2}^{c} e^{\beta u x} d x, u \in\{0,1\}, \\
\ln \left(\hat{S}(c \mid u) / \hat{S}\left(c+c_{n} \mid u\right)\right)=h_{3} \int_{c}^{c+c_{n} / 2} e^{\beta u x} d x+h_{4} \int_{c+c_{n} / 2}^{c+c_{n}} e^{\beta u x} d x, u \in\{0,1\},
\end{gathered}
$$

though the solutions to $h_{i}$ 's may not be nonnegative. However, for each $\beta$ in a neighbourhood of the GMLE $\hat{\beta}$ defined in part a, there exists a positive solution to $\left(h_{1}, \ldots, h_{4}\right)$ to the four equations (corresponding to $u \in\{0,1\})$ in $(3.8)$ and $(3.9)$ and thus, there exist many proper solutions to $\left(\beta, h_{1}, \ldots, h_{4}\right)$. Each of such $\left(\beta, h_{1}, h_{2}, h_{3}, h_{4}\right)$ maximizes $\mathcal{L}_{o}$. Consequently, this approach is not ideal, though it can lead to a consistent estimator of $\beta$.

d. An SMLE approach. In view of $(3.9)$, if $c_{n} \approx 0$, we have

$$
\ln \left(\hat{S}(c \mid u) / \hat{S}\left(c+c_{n} \mid u\right)\right) \approx e^{\beta u c}\left(h_{3} \int_{c}^{c+c_{n} / 2} d x+h_{4} \int_{c+c_{n} / 2}^{c+c_{n}} d x\right), u \in\{0,1\}
$$

$h_{3}+h_{4}$ and $\beta$ can be uniquely determined by the two equations in (3.10). Thus the parameters are really $h_{1}, h_{2}, h_{3}+h_{4}$ and $\beta$ and the degree of freedom in the second naive approach reduces to 4 . If we revise (3.8) as follows,

$$
-\ln (\hat{S}(c \mid u))=h_{1} \int_{0}^{c / 2} e^{\beta u c / 2} d x+h_{2} \int_{c / 2}^{c} e^{\beta u c} d x, u \in\{0,1\},
$$

which uniquely determines $h_{1}$ and $h_{2}$, the four parameters, $S\left(c+c_{n} \mid u\right)$ and $S\left(c+c_{n} \mid u\right)$ for $u \in\{0,1\}$, can be reparametrized equivalently as $\beta$ and $h_{o}$ which is piecewise constant on $(0, c / 2],(c / 2, c]$ and $\left(c, c+c_{n}\right]$ with values $h_{1}, h_{2}$ and $h_{3}$, respectively (notice that the new parameter is $\left(\beta, h_{1}, h_{2}, h_{3}\right)$, with 4 degrees of 
freedom again). Recall the generalized likelihood

$$
\begin{aligned}
\mathcal{L}_{o}= & \left(1-\exp \left(-\int_{0}^{c} e^{\beta x} h_{o}(x) d x\right)\right)^{n_{11}}\left[\exp \left(-\int_{0}^{c} e^{\beta x} h_{o}(x) d x\right)\left(1-\exp \left(-\int_{c}^{c+c_{n}} e^{\beta x} h_{o}(x) d x\right)\right)\right]^{n_{21}} \\
& \times\left(\exp \left(-\int_{0}^{c+c_{n}} e^{\beta x} h_{o}(x) d x\right)\right)^{n_{31}}\left(1-\exp \left(-0.5\left(c h_{1}+c h_{2}\right)\right)\right)^{n_{10}} \\
& \times\left(\exp \left(-0.5\left(c h_{1}+c h_{2}\right)\right)\left(1-\exp \left(-\left(c_{n} h_{3}\right)\right)\right)\right)^{n_{20}}\left(\exp \left(-\left(0.5 c h_{1}+0.5 c h_{2}+c_{n} h_{3}\right)\right)\right)^{n_{30}} .
\end{aligned}
$$

Replacing $e^{\beta u x} h_{o}(x)$ by a piecewise constant, say

$$
h_{1} e^{\beta u c_{2} /} \mathbf{1}\left(x \in(0, c / 2]+h_{2} e^{\beta u c} \mathbf{1}\left(x \in(c / 2, c]+h_{3} e^{\beta u c+c_{n} / 2} \mathbf{1}\left(x \in\left(c, c+c_{n}\right]\right. \text {, }\right.\right.
$$

$\mathcal{L}_{o}$ becomes

$$
\begin{aligned}
\mathcal{L}= & \left(1-\exp \left(-\int_{0}^{c / 2} e^{\beta c / 2} h_{1} d x-\int_{c / 2}^{c} e^{\beta c} h_{2} d x\right)\right)^{n_{11}} \\
& \times\left[\exp \left(-\int_{0}^{c / 2} e^{\beta c / 2} h_{1} d x-\int_{c / 2}^{c} e^{\beta c} h_{2} d x\right)(\underbrace{\left.\left.1-\exp \left(-\int_{c}^{c+c_{n}} e^{\beta c} h_{3} d x\right)\right)\right]^{n_{21}}}_{\approx h_{3} e^{\beta c} c_{n}}\right. \\
& \times\left(\exp \left(-\int_{0}^{c+c_{n}} e^{\beta c} h_{3} d x\right)\right)^{n_{31}}\left(1-\exp \left(-0.5\left(c h_{1}+c h_{2}\right)\right)\right)^{n_{10}} \\
& \times\left(\exp \left(-0.5\left(c h_{1}+c h_{2}\right)\right)(\underbrace{1-\exp \left(-\left(c_{n} h_{3}\right)\right)}_{\approx h_{3} c_{n}})\right)^{n_{20}\left(\exp \left(-\left(0.5 c h_{1}+0.5 c h_{2}+c_{n} h_{3}\right)\right)\right)^{n_{30}} .}
\end{aligned}
$$

It can be verified that for $\left(\beta, h_{1}, h_{2}, h_{3}\right)$, there is a different vector $\left(\beta, h_{1}, h_{2}^{*}, h_{3}^{*}\right)$ such that $\mathcal{L}_{o}\left(\beta, h_{1}, h_{2}, h_{3}\right)=$ $\mathcal{L}\left(\beta, h_{1}, h_{2}^{*}, h_{3}^{*}\right)$. Thus, one can take $\mathcal{L}$ as the semi-parametric likelihood and the SMLE of $\beta$ (and $\left.h_{3}\right)$ can be solved through the GMLE $\hat{S}(\cdot \mid \cdot)$, which leads to $\tilde{\beta}=\frac{1}{c} \ln \frac{\frac{n_{21}}{n_{1}-n_{21}}}{\frac{n_{20}}{n_{0}-n_{20}}}$, which is the same as the GMLE of $\beta$ in (3.7). Notice that replacing $e^{\beta u x} h_{o}(x)$ by a different piecewise constant such as

$$
\begin{aligned}
& h_{1} e^{\beta u 0} \mathbf{1}\left(x \in(0, c / 2]+h_{2} e^{\beta u c / 2} \mathbf{1}\left(x \in(c / 2, c]+h_{3} e^{\beta u c} \mathbf{1}\left(x \in\left(c, c+c_{n}\right)\right. \text { or }\right.\right. \\
& h_{1} e^{\beta u c} \mathbf{1}\left(x \in(0, c / 2]+h_{2} e^{\beta u c} \mathbf{1}\left(x \in(c / 2, c]+h_{3} e^{\beta u c} \mathbf{1}\left(x \in\left(c, c+c_{n}\right],\right.\right.\right.
\end{aligned}
$$

the limit of the SMLE will be the same. Notice that $J=2$ (see $(3.3)$ ) here, as $u \sim \operatorname{bin}(1,0.5)$. This case also motivates Eq. (3.3) and $\mathcal{L}$ in $\S 3.2$. This concludes Example 3.1.

Remark 5. The discussion in Example 3.1 suggests that if $u \in\{0,1\}$, then one can get a GMLE of $\beta$ based on $\mathcal{L}_{*}$ in $(2.1)$ as follows.

1. First obtain the GMLE of $S_{o}$ and $S(\cdot \mid 1)$, based on the samples with $u_{i}=0$ and $u_{i}=1$, respectively, by the self-consistent algorithm (see Turnbull (1976)).

2. Let $\hat{\beta}=\frac{1}{m} \sum_{j=1}^{m} \frac{1}{b_{k_{j}}-a} \ln \frac{\ln \left(S\left(b_{k_{j}} \mid 1\right) / S\left(a_{k_{j}} \mid 1\right)\right)}{\ln \left(S\left(b_{k_{j}} \mid 0\right) / S\left(a_{k_{j}} \mid 0\right)\right)}$, where $\left(a_{k_{j}}, b_{k_{j}}\right], j=1, \ldots, m$, are all the II's that satisfy $b_{k_{j}}-b_{k_{j}-1} \approx 0, a_{k_{j}}>a$, and $S\left(b_{k_{j}} \mid u\right)>S\left(a_{k_{j}} \mid u\right)$ for $u \in\{0,1\}$.

If the covariate $u$ takes on finitely many values, the approach is applicable after minor modifications. The drawback of this approach is that it does not work if there are very few ties in the covariate $u_{i}$ 's.

Remark 6. If $\tau<\infty$ and $\sup \mathcal{S}_{F_{L}}<\infty$, then $b_{m}<\infty$, where $b_{m}$ is the largest among the right-end points of the innermost intervals. If one defines $h_{o}$ to be piecewise constant on the II's, then such $h_{o}$ does not lead to a proper survival function. However it is seen from Example 3.1 that there is no need to define $h_{o}$ on the interval $\left(a_{m}, b_{m}\right]$ in the likelihood function $\mathcal{L}$, as long as $S_{o}\left(b_{m}\right)=0$. In the latter case, the likelihood will remain the same for any reasonable definition of $h_{o}$ on $\left[a_{m}, b_{m}\right]$.

\section{Simulation Studies}

The next table presents the simulation results for the naive estimator $\breve{\beta}$ and the SMLE $\hat{\beta}$ under the assumption in Example 3.1. The simulation results suggest that $\hat{\beta}$ is consistent, but not $\breve{\beta}$.

We carried out simulation studies under the mixed case IC model. The mixed case IC model is implemented by $\left(L_{i}, R_{i}\right)=\left(W_{i-1}, W_{i}\right)$ if $Y \in\left(W_{i-1}, W_{i}\right]$, where $W_{0}=0, W_{i}=i V, i \geq 1, V$ is from 
Table 1. Simulation Results for the naive estimator $\check{\beta}$ and the SMLE $\hat{\beta}$

\begin{tabular}{cccccc}
\hline sample size & $\beta$ & $\breve{\beta}$ & $s d_{\hat{\beta}}$ & $\hat{\beta}$ & $s d_{\hat{\beta}}$ \\
\hline 2000 & 0.5 & 0.284 & 0.086 & 0.564 & 0.299 \\
20000 & 0.5 & 0.266 & 0.031 & 0.519 & 0.162 \\
2000000 & 0.5 & 0.259 & 0.009 & 0.493 & 0.086 \\
\hline
\end{tabular}

$U(0,0.4)$. In addition if there is a fixed II $(b, c]$, then $L_{i}=a$ if $a \leq L_{i} \leq b$ and $R_{i}=b$ if $a \leq R_{i} \leq b$. We carried out simulation under the following setups.

(1) $\mathcal{S}_{F_{L}} \cup \mathcal{S}_{F_{R}}$ is dense in $(c, \tau) . u \sim U[0,1)$, in addition to $u \sim \operatorname{bin}(1,0.5)$.

(2) A population II is $(b, c]$, where $a<b<c$.

(3) A population II is $(b, c]$, where $b<a<c$.

We generated data with 5000 replications each for sample sizes $\mathrm{n}=100,200$ and 400 . The cut point is 0.5 and $\lambda=0.2$. Table 2 displays the results. Our simulation study suggests that the SMLE $\hat{\beta}$ is consistent and the convergence rate is $n^{\frac{1}{2}}$.

Table 2. Simulation Results for the SMLE

\begin{tabular}{cccccccc}
\hline sample size & $\beta$ & $h_{o}$ & $a$ & $u$ & $(a, b)$ & $\hat{\beta}$ & $S D_{\hat{\beta}}$ \\
\hline 100 & 0.5 & 1 & 0.2 & $\operatorname{bin}(1,0.5)$ & $(0.3,0.8)$ & 0.512 & 0.238 \\
200 & 0.5 & 1 & 0.2 & $\operatorname{bin}(1,0.5)$ & $(0.3,0.8)$ & 0.516 & 0.183 \\
400 & 0.5 & 1 & 0.2 & $\operatorname{bin}(1,0.5)$ & $(0.3,0.8)$ & 0.489 & 0.111 \\
\hline 100 & 0.5 & 1 & 0.2 & $U(0,1)$ & $(0.3,0.8)$ & 0.542 & 0.423 \\
200 & 0.5 & 1 & 0.2 & $U(0,1)$ & $(0.3,0.8)$ & 0.491 & 0.298 \\
400 & 0.5 & 1 & 0.2 & $U(0,1)$ & $(0.3,0.8)$ & 0.469 & 0.196 \\
\hline 100 & 1 & $\exp (\lambda t)$ & 0.5 & $U(0,1)$ & $(0.4,0.6)$ & 1.243 & 0.897 \\
200 & 1 & $\exp (\lambda t)$ & 0.5 & $U(0,1)$ & $(0.4,0.6)$ & 1.132 & 0.612 \\
400 & 1 & $\exp (\lambda t)$ & 0.5 & $U(0,1)$ & $(0.4,0.6)$ & 1.072 & 0.386 \\
\hline 100 & 1 & $\exp (\lambda t)$ & 0.5 & $\operatorname{bin}(1,0.5)$ & $(0.4,0.6)$ & 1.072 & 0.532 \\
200 & 1 & $\exp (\lambda t)$ & 0.5 & $\operatorname{bin}(1,0.5)$ & $(0.4,0.6)$ & 1.074 & 0.296 \\
400 & 1 & $\exp (\lambda t)$ & 0.5 & $\operatorname{bin}(1,0.5)$ & $(0.4,0.6)$ & 1.025 & 0.204 \\
\hline 100 & 1 & $\exp (\lambda t)$ & 0.5 & $\operatorname{bin}(1,0.5)$ & $(0.6,0.8)$ & 1.057 & 0.534 \\
200 & 1 & $\exp (\lambda t)$ & 0.5 & $\operatorname{bin}(1,0.5)$ & $(0.6,0.8)$ & 1.040 & 0.302 \\
400 & 1 & $\exp (\lambda t)$ & 0.5 & $\operatorname{bin}(1,0.5)$ & $(0.6,0.8)$ & 1.016 & 0.221 \\
\hline 100 & 2 & $\exp (\lambda t)$ & 0.5 & $U(0,1)$ & $(0.6,0.8)$ & 2.178 & 0.651 \\
200 & 2 & $\exp (\lambda t)$ & 0.5 & $U(0,1)$ & $(0.6,0.8)$ & 2.114 & 0.523 \\
400 & 2 & $\exp (\lambda t)$ & 0.5 & $U(0,1)$ & $(0.6,0.8)$ & 2.058 & 0.359 \\
\hline
\end{tabular}




\section{$5 \quad$ Concluding Remark}

Even though we only consider case that the covariate is of the form $\mathbf{z}(t)=(t-a) \mathbf{u} \mathbf{1}(t \geq a)$, the result can be generalized to the case of other time-dependent covariates, such as the form $\mathbf{z}(t)=\mathbf{u} g(t)$, where $\mathbf{u}$ is a covariate and $g(t)$ is a function. For instance, the two modification are trivially applicable to the case that $g(t)=\mathbf{1}(t \geq a)$, though (3.3) is not necessary. However, if $g(t)=(t-a)^{2}$ then the modification in $(3.3)$ is necessary and works.

Acknowledgments. The authors thank the editor and a referee for their invaluable comments.

\section{References}

1. D.R. Cox, "Regression models and life tables," Journal of Royal Statistics Society, B, vol. 34, pp. 187-220, 1972 .

2. D.R. Cox, and D. Oakes, "Analysis of Survival Data," Chapman \& Hall, 1984.

3. K. Leffondre, M. Abrahamowicz, and J. Siemiatycki, "Evaluation of Cox's model and logistic regression for matched case-control data with time-dependent covariates: a simulation study," Statistics in Medicine, vol. 22, pp. 3781-3794, 2003.

4. K. Leffondre, W. Wynant, Z. Cao, et al. "A weighted Cox model for modeling time-dependent exposures in the analysis of case-control studies," Statistics in Medicine, vol. 29, pp. 839-850, 2010.

5. T. Masaaki, and S. Masato, "Analysis of survival data having time-dependent covariates," IEEE Transaction on Neural Networks, vol. 20, pp. 389-394, 2009.

6. R.W. Platt, K. S. Joseph3, C. V. Ananth, J. Grondines1, M. Abrahamowicz, and M. S. Kramer, "A proportional hazards model with time-dependent covariates and time-varying effects for analysis of fetal and infant death," American Journal of Epidemiology, vol. 160, pp. 199-206, 2004.

7. A. Schick, and Q.Q. Yu, "Consistency of the GMLE with mixed case interval-censored data," Scandinavian Journal of Statistics, vol. 27 pp. 45-55, 2000.

8. L. Stephan, and S. Michael, "Parsimonious analysis of time-dependent effects in the Cox model," Statistics in Medicine, vol. 26, pp. 2686-2698, 2007.

9. T. Therneau, and P. Grambsch, "Modeling survival data : extending the Cox model," Springer. 2000.

10. B. W. Turnbull, "The empirical distribution function with arbitrary grouped, censored and truncated data," Journal of Royal Statistics Society, B, vol. 38, pp. 290-295, 1976.

11. G.Y.C. Wong, Q.G. Diao, and Q.Q. Yu, "Piecewise proportional hazards models with interval-censored data," Journal of Statistical Computation and Simulation, vol. 88, pp.140-155, 2018.

12. G. Y. C. Wong, M. P. Osborne, Q.G. Diao, and Q.Q. Yu, Piece-wise Cox Models With Right-Censored Data," Communications in Statistics - Simulation and Computation, vol. 46, pp. 7894-7908, 2017.

13. G.Y.C. Wong, and Q.Q. Yu, "Estimation under the Lehmann regression model with interval-censored data," Communications in Statistics - Simulation and Computation, 41 1489-1500, 2012.

14. H.M. Zhang, and C.F. Huang, "Nonparametric survival analysis on time-dependent covariate effects in case-cohort sampling design," Statistica Sinica, vol. 16, pp. 267-285. 2006.

15. M. Zhou, "Understanding the Cox regression models with time-change covariates," American Statistian, vol. 55, pp. 153-155, 2001. 